ASTROPHYSICAL JOURNAL, ACCEPTED FOR PUBLICATION

Preprint typeset using $\mathrm{LT}_{\mathrm{E}} \mathrm{X}$ style emulateapj v. 10/10/03

\title{
ASTEROSEISMIC SIGNATURES OF SMALL CONVECTIVE CORES
}

\author{
M. S. CUNHA ${ }^{1}$ \\ Centro de Astrofísica da Universidade do Porto, Rua das Estrelas, 4150-Porto, Portugal \\ AND \\ T. S. METCALFE \\ High Altitude Observatory and Scientific Computing Division, NCAR, PO Box 3000, Boulder, CO 80307 USA \\ Astrophysical Journal, accepted for publication
}

\begin{abstract}
We present an analytical study of the effect of small convective cores on the oscillations of solar-like pulsators. Based on an asymptotic analysis of the wave equation near the center of the star, we derive an expression for the perturbations to the frequencies of radial modes generated by a convective core and discuss how these perturbations depend on the properties of the core. Moreover, we propose a diagnostic tool to isolate the predicted signature of the core, constructed from a particular combination of the oscillation frequencies, and we validate this tool with simulated data. We also show that the proposed tool can be applied to the pulsation data soon expected from satellite missions such as CoRoT and Kepler to constrain the amplitude of the discontinuity in the sound speed at the edge of the convective core, the ratio between the sound speed and the radius at this same location, and the stellar age.
\end{abstract}

Subject headings: convection-methods: analytical—stars: interiors—stars: oscillations

\section{INTRODUCTION}

Space-based asteroseismology began in 1999, when the $5 \mathrm{~cm}$ star tracker mounted on the Wide-field InfraRed Explorer (WIRE) satellite was used to detect oscillations in the $\mathrm{K}$ giant $\alpha$ UMa (Buzasi et al. 2000), and this instrument has continued to produce a steady stream of asteroseismic data (e.g., see Bruntt et al. 2007). The $15 \mathrm{~cm}$ Micro-variability \& Oscillations of Stars (MOST) satellite was launched in 2003, and was the first satellite in orbit that was actually designed for asteroseismology (Walker et al. 2003). Although MOST's failure to detect solar-like oscillations in Procyon (Matthews et al. 2004) was later traced to a larger than expected source of non-white instrumental noise (Bedding et al. 2005), its ability to conduct nearly uninterrupted time-series photometry for durations of up to two months has produced exquisite data for pulsating stars across the H-R diagram (e.g., see Randall et al. 2005; Aerts et al. 2006; Saio et al. 2006; Rowe et al. 2006; Cameron et al. 2007).

The next era of space-based asteroseismology began with the successful launch of the $27 \mathrm{~cm}$ Convection, Rotation and planetary Transits (CoRoT) satellite in December 2006 (Baglin et al. 2006), and will continue with the anticipated launch of the $95 \mathrm{~cm}$ Kepler satellite in 2008 (Christensen-Dalsgaard et al. 2007). These missions promise advances in the study of solar-like oscillations comparable to what WIRE and MOST have achieved for the larger amplitude classical pulsators. With the frequency precision expected to approach $\sim 0.1 \mu \mathrm{Hz}$ from this next generation of satellites, we can begin to consider the possible detection of extremely subtle asteroseismic signals.

There is a long history of constructing combinations of the observed oscillation frequencies to extract useful asteroseismic information. The simplest such combination is the average frequency spacing between modes of consecutive radial order but the same spherical degree-the large separation-

\footnotetext{
${ }^{1}$ Visiting Scientist, High Altitude Observatory, NCAR, PO Box 3000, Boulder, CO 80307 USA

Electronic address: mcunha@astro.up.pt, travis@ hao.ucar.edu
}

which provides an estimate of the mean stellar density (Cox 1980). Similarly, the average spacing between modes of consecutive radial order but with spherical degrees that differ by 2 - the small separation-is sensitive to chemical gradients in the deep interior, tracing the stellar evolutionary state (Dappen et al. 1988).

The idea that sharp variations in the internal structure of distant stars generate signatures in the frequencies of low degree modes that can be recognized and interpreted with the appropriate seismic tools has been explored in a number of papers. In this context, theoretical derivations were carried out of the signal expected from sharp variations taking place at the boundary of convective envelopes (Monteiro et al. 2000) and in the region of helium ionization (Monteiro \& Thompson 1998; Houdek \& Gough 2007). Moreover, the expected signature of convective cores on the small frequency separations has also been considered by Roxburgh \& Vorontsov (2001). However, all of these works assumed that the sharp variation in the structure under investigation was located far from the turning points of the oscillation modes, i.e., well within their propagation cavities. Consequently, the analyses carried out in these works do not directly apply to studies of small convective cores, such as those present in main-sequence solarlike pulsators.

Attempts to identify and interpret in a systematic manner the signatures of convective cores in solar-like pulsators directly from simulated data have recently been carried out by Mazumdar et al. (2006). Based on an analysis of the results obtained from simulated data, the authors suggested a diagnostic tool to estimate the masses of the convective cores and the stellar ages. While recognizing the importance of studying simulated data, the chances of constructing the optimal diagnostic tool to detect and characterize small convective cores may be greater if we know what signal to expect. Moreover, a theoretical understanding of the expected signal can provide us with a direct link between the structure under investigation and the observations-moving us toward our goal of finding the best way to infer information about that structure. With this in mind, in this paper 
we derive the expected signal of a small convective core on the oscillation frequencies of solar-like pulsators. Unlike Monteiro et al. (2000), Roxburgh \& Vorontsov (2001), and Houdek \& Gough (2007), we do not assume that the region of sharp structural variation, which in the present case is the edge of the convective core, is placed well within the propagation region of the modes. In fact, the analysis is focused on the opposite case, in which the edge of the convective core is sufficiently close to the center to affect the radial modes more significantly than the modes with degree $\ell \geq 1$.

We derive the theoretical expression for the signature of a small convective core in $\$ 2.1$. In $\$ 2.2$ we construct a diagnostic tool, based on a particular combination of the frequencies, to isolate this signature. In $\$ 3$ we validate the theoretical predictions and the diagnostic tool using simulated data from a series of $1.3 \mathrm{M}_{\odot}$ models. Moreover, we discuss the successes and limitations of the proposed tool, as well as the information that it can reveal. Finally, in $\$ 4$ we outline the potential for future work in this area, and we discuss possible observational tests.

\section{SIGNATURE OF A CONVECTIVE CORE}

\subsection{Theoretical expectation}

Let $\boldsymbol{\xi}=(\xi, 0,0) r e^{-i \omega t}$ be the displacement, defined with respect to a spherical coordinate system $(r, \theta, \phi)$, for radial, adiabatic, acoustic oscillations in a spherically symmetric star, with $\omega$ the oscillation frequency and $t$ the time. Through the appropriate mathematical manipulation of the equations of motion and mass conservation for radial pulsations, under the adiabatic approximation, the dimensionless displacement $\xi$ is found to obey the second order differential equation (e.g. Gough 1993),

$$
\frac{\mathrm{d}}{\mathrm{d} r}\left(\gamma p r^{4} \frac{\mathrm{d} \xi}{\mathrm{d} r}\right)+\left\{r^{3} \frac{\mathrm{d}}{\mathrm{d} r}[(3 \gamma-4) p]+r^{4} \rho \omega^{2}\right\} \xi=0,
$$

where $\gamma$ is the first adiabatic exponent, $p$ is the pressure and $\rho$ is the density. By defining new dependent and independent variables, $\Xi=r_{\text {ref }}^{1 / 2} r^{3 / 2} \rho^{1 / 2} c \xi$ and $z=\ln \left(r / r_{\text {ref }}\right)$ respectively, where $c=\sqrt{\gamma p / \rho}$ is the sound speed and $r_{\text {ref }}$ is a fiducial value of $r$, and substituting into Eq. (1), the latter reduces to the standard form,

$$
\frac{\mathrm{d}^{2} \Xi}{\mathrm{d} z^{2}}+K^{2} \Xi=0
$$

where

$$
K^{2}=\frac{\omega^{2}-\omega_{\mathrm{c}}^{2}}{c^{2}} r^{2}-\frac{1}{4},
$$

and $\omega_{\mathrm{c}}$ is a critical acoustic frequency (e.g., see Gough 1993, Eq. 4.8.7). Since our aim is to study the effect of a small convective core on the eigenfrequencies, we are particularly interested in the form of $\omega_{c}$ close to the center of the star. From the general expression for $\omega_{\mathrm{c}}$, we find that when $r \rightarrow 0$, $\omega_{\mathrm{c}}^{2} \approx 2 c^{2} / r^{2}$. Using this expression for $\omega_{\mathrm{c}}^{2}$, we then find the critical frequency $\omega_{\mathrm{c}}^{*}$ at which $K^{2}=0$ to be,

$$
\omega_{\mathrm{c}}^{*} \approx \frac{3 c}{2 r} .
$$

A model with a small convective core exhibits a sharp structural variation close to the inner turning point of Eq. (2). This sharp variation can be seen in the sound speed profiles of main-sequence models with mass $M=1.3 \mathrm{M}_{\odot}$, shown in Figure 1 for a range of stellar ages. Our aim is to determine

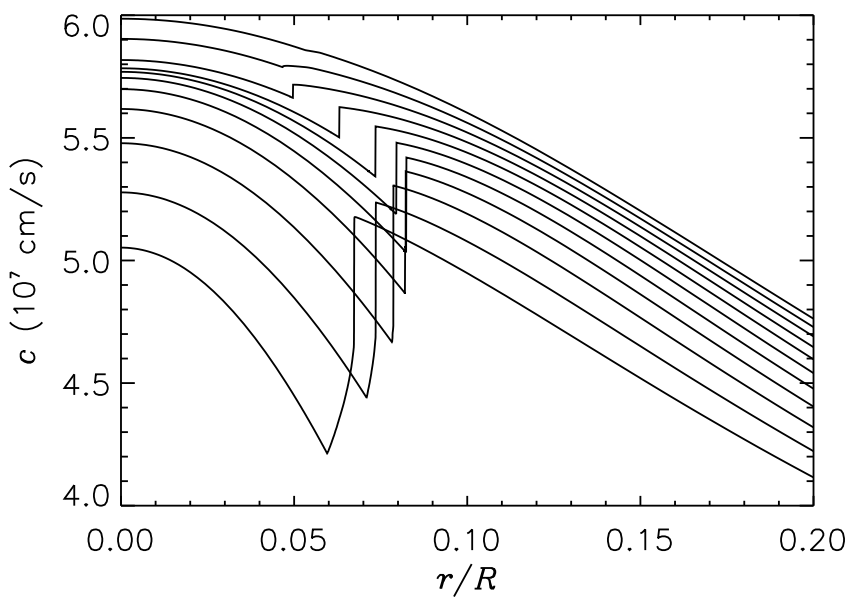

FIG. 1.- Sound speed in the inner layers of main-sequence models with $M=1.3 \mathrm{M}_{\odot}$, and ages ranging from $0.25 \mathrm{Gyr}$ (top curve) to $5.25 \mathrm{Gyr}$ (bottom curve) in intervals of 0.5 Gyr. The discontinuity in $c$ marks the edge of the convective core, which expands during the evolution of the star up to an age of $3.25 \mathrm{Gyr}$ and then begins to contract.

how the eigenfrequencies of an otherwise similar model with a smooth structure around the edge of the convective corehereafter the unperturbed model - are modified by the presence of this sharp variation.

To achieve this we must find an expression for $\Xi$ that is valid close to the inner turning point of Eq. (2). Such an expression for $\Xi$ is derived in Appendix A. In short, we define new dependent and independent variables and substitute them into Eq. (2) to obtain an equation that resembles the Airy equation with an additional term (cf. Eq. A3 in Appendix A). Applying Olver's comparison method (Olver 1974) we then find, close to the inner turning point,

$$
\Xi \approx a|x|^{1 / 4}|K|^{-1 / 2}\left[A_{\mathrm{i}}(0)-A_{\mathrm{i}}^{\prime}(0) x\right],
$$

where $a$ is a constant related to the normalization of eigenfunctions, $A_{\mathrm{i}}(0)$ and $A^{\prime}{ }_{\mathrm{i}}(0)$ are the values taken at $x=0$ of the first solution to the Airy equation and its first derivative respectively, and $x$ is the new independent variable, which is related to $z$ through Eq. A1 in Appendix A In deriving Eq. (5) we have neglected terms of $\mathcal{O}\left(x^{3}\right)$. So, this equation is approximately correct when $x$ is sufficiently close to 0 . Note that unlike $r$, the independent variable $x$ depends on the value of the eigenfrequency. For a given frequency it goes through 0 at the inner turning point, and is negative when $K^{2}<0$ and positive when $K^{2}>0$. The relation between $x$ and $r$ for different values of the eigenfrequency $\omega$, as well as the regions where $K^{2}<0$ and $K^{2}>0$, are shown in Figure 2 for an unperturbed stellar model with $M=1.3 \mathrm{M}_{\odot}$. The unperturbed models were obtained by fitting the sound speed profiles of each stellar model with smooth functions around the edge of the convective core. The density profile was then derived by assuming hydrostatic equilibrium.

To study the effect of a small convective core on the oscillation frequencies, we start from an integral equation for the frequency, which can be derived from Eq. (1) after multiplying by $\xi$ and integrating by parts between $r=0$ and $r=R$, where $R$ is the radius at the stellar surface. The resulting well known equation is,

$$
\omega^{2}=\frac{I_{2}-\mathcal{B}}{I_{1}}
$$


where

$$
\begin{aligned}
& I_{2}=\int_{0}^{R}\left[\gamma p r^{4}\left(\frac{d \xi}{d r}\right)^{2}-r^{3} \frac{d}{d r}[(3 \gamma-4) p] \xi^{2}\right] d r, \\
& I_{1}=\int_{0}^{R}\left[\rho r^{4} \xi^{2}\right] d r
\end{aligned}
$$

and

$$
\mathcal{B}=\mathcal{B}(R)-\mathcal{B}(0)=\left[\gamma p r^{4} \xi \frac{d \xi}{d r}\right]_{r=0}^{r=R} .
$$

When the oscillations are below the acoustic cutoff frequency, the surface term $\mathcal{B}$ can safely be neglected. In fact, it follows from the regularity condition at $r=0$ that $\mathcal{B}(0)=0$ and, given the small value of $p(R)$, if the oscillations are evanescent in the outer layers $\mathcal{B}(R)$ will also be small.

We now consider the above mentioned unperturbed smooth model and an otherwise similar model with a sharp structural variation at the edge of a small convective core-hereafter the perturbed model. If $\mathcal{B}$ is neglected in Eq. (6), the difference between the oscillation frequencies in the perturbed and unperturbed models in a linear approximation is given by

$$
\delta \omega=\frac{\delta I_{2}-\omega^{2} \delta I_{1}}{2 \omega I_{1}}
$$

where all unperturbed quantities refer to the smooth model. Equation (6) with $\mathcal{B}$ neglected constitutes a variational principle for the frequency $\omega$ (e.g. Chandrasekhar 1964; Gough 1993). Thus, to first order the perturbation to the eigenfunctions does not contribute to the perturbation to the eigenfrequencies and we can write,

$$
\delta I_{1}=\int_{0}^{R} \delta \rho r^{4} \xi^{2} \mathrm{dr}
$$

and

$$
\begin{aligned}
\delta I_{2}= & \int_{0}^{R}\left\{r^{4} \delta(\gamma p)\left(\frac{\mathrm{d} \xi}{\mathrm{d} r}\right)^{2}\right. \\
& \left.-r^{3} \frac{\mathrm{d}}{\mathrm{d} r}[3 \delta(\gamma p)-4 \delta p] \xi^{2}\right\} \mathrm{d} r
\end{aligned}
$$

where $\delta \rho, \delta p$ and $\delta \gamma$ denote perturbations at fixed radius. To obtain an expression for $\delta \omega$ as a function of $\omega$, we manipulate Eqs. (7, 9) in a way very similar to that presented in Monteiro et al. (1994). In particular, Eqs. (8) and (9) are written in terms of the perturbations $\delta(\gamma p), \delta c^{2}$ and $\delta g$, with $g=-\frac{1}{\rho} \frac{\mathrm{d} p}{\mathrm{~d} r}$ and then integrated by parts (see Appendix $\mathrm{B}$ for details). As discussed in Appendix [B, the perturbation to the eigenfrequencies is determined predominantly by the terms associated with $\delta c^{2}$. Describing this perturbation with a modified step function, we find (cf. Eq. B10)

$$
\begin{aligned}
& \delta \omega \approx- \frac{A_{\delta}}{2 \omega I_{1}}\left[\frac{|K| r^{3} \rho c^{2} \xi^{2}}{|x|^{1 / 2}}\right]_{x=0}\left[\frac{r\left(4 g+\omega^{2} r\right)}{c^{2}|K|^{2}}\right]_{x=x_{\mathrm{d}}} \\
& \times\left[|x| x-\frac{A_{\mathrm{i}}^{\prime}(0)}{A_{\mathrm{i}}(0)}|x| x^{2}+\frac{1}{3} \frac{A_{\mathrm{i}}^{\prime}(0)^{2}}{A_{\mathrm{i}}(0)^{2}}|x| x^{3}\right]_{x=x_{\mathrm{d}}},
\end{aligned}
$$

where $A_{\delta}$ is a positive constant related to the sound speed increase at the edge of the convective core and $x_{\mathrm{d}}$ is the value taken by the independent variable $x$ at the location of the edge of the convective core. Both $x$ and $x_{\mathrm{d}}$ are functions of frequency. Through this dependence, Eq. (10) expresses how the

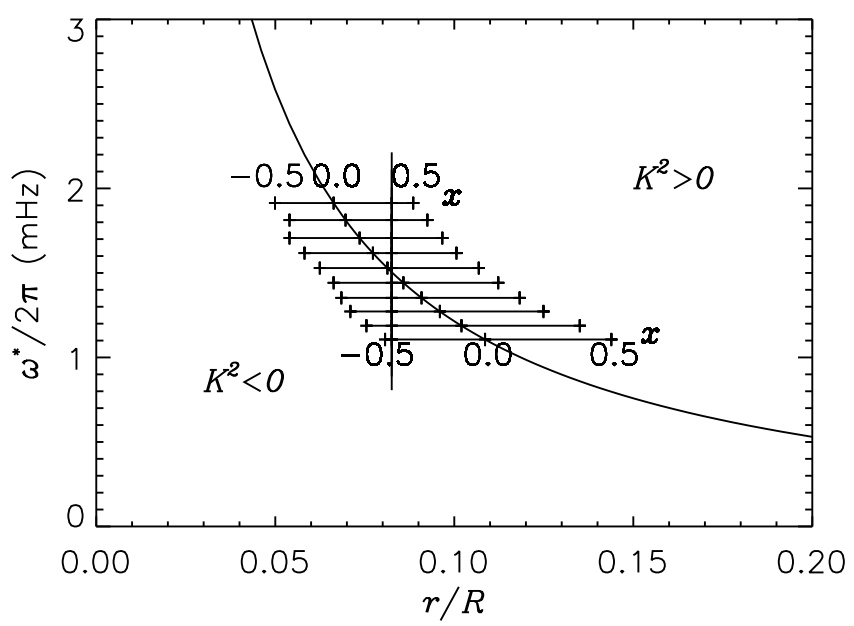

FIG. 2.- The full curve shows the critical frequency obtained by imposing $K^{2}=0$ for the inner layers of an unperturbed main-sequence model with $M=1.3 \mathrm{M}_{\odot}$ at the age when its convective core reaches maximum size $(3.25 \mathrm{Gyr})$. For each frequency, this curve gives the maximum propagation depth of a radial mode when the eigenfunctions are the solutions to Eq. 2]. The vertical line shows the radial position of the edge of the convective core. The horizontal lines are axes for the independent variable $x$ in the range $[-0.5,0.5]$. These are shown for eigenmode frequencies of the corresponding perturbed model, with radial orders ranging from $n=11$ (bottom line) to $n=20$ (top line).

frequency perturbation is modulated with frequency. According to this equation the frequency perturbation goes through zero when $x_{\mathrm{d}}=0$, i.e. when the frequency is such that the turning point and the edge of the convective core are located at the same depth.

If the signal anticipated by Eq. (10) could be detected in the oscillation frequencies of real stars, then information about the size and position of the sound speed discontinuity at the edge of the convective core could be inferred from the amplitude $A_{\delta}$ and the frequency at which $\delta \omega=0$, respectively. With this in mind, in the next section we present a diagnostic tool that will allow us to isolate the signal derived above.

\subsection{Diagnostic tool}

When deriving Eq. (10) we assumed that our model differs from a smooth model only at the edge of the convective core. However, sharp variations in the internal structure of a star are known to occur also at other locations, such as the base of the outer convective region and the region of helium ionization. These, in turn, will generate additional perturbations to the eigenfrequencies, as compared with those of a smooth model (e.g., Monteiro et al. 1994; Basu 1997; Monteiro \& Thompson 2005; Houdek \& Gough 2007). Thus, to isolate the signal produced by the edge of a convective core, we must combine the oscillation frequencies of real stars in such a way as to cancel out, as much as possible, the signal coming from all other sources. With this in mind, we write the frequency $\nu_{n, \ell}=\omega_{n, \ell} / 2 \pi$ of a mode with radial order $n$ and degree $\ell$ in a given star as the sum of three components

$$
\nu_{n, \ell}=\nu_{n, \ell}^{s}+\delta \nu_{n, \ell}^{p}+\delta \nu_{n, \ell}^{c},
$$

where $\nu_{n, \ell}^{s}$ is the frequency of the mode in the smooth model, $\delta \nu_{n, \ell}^{p}$ is the perturbation to that frequency arising from all sharp variations in the structure of the star taking place well within the propagation region of the same mode (which we shall consider to be of low degree, with $\ell \leq 3$ ), and $\delta \nu_{n, \ell}^{c}$ is 
the frequency perturbation produced by the edge of the small convective core.

While sharp variations in the envelope of a star will affect the frequencies of all modes of low degree, the discontinuity in the sound speed at the edge of a small convective core for a star slightly more massive than the Sun might be expected to affect primarily the frequencies of radial modes. This is not to say that the frequencies of modes of degree $\ell=1$, which also propagate to very deep layers, will not be affected at all by the convective core. In fact, to correctly derive the expected signal for a dipolar mode, the Cowling approximation should be avoided. The derivation should then be started from the formalism recently presented by Takata (2006), which shows that the inner turning point of dipolar modes is modified when the equations are treated without neglecting the perturbation to the gravitational potential. However, such an analysis is beyond the scope of the present paper. Hence, based on the idea that radial modes are most sensitive to the deepest layers of a star, we will proceed by assuming $\delta \nu_{n, \ell}^{c} \approx 0$ for modes with $\ell \geq 1$. This assumption will be tested in the following section. Moreover, given that we are interested in modes with degree $\ell \leq 3$, we neglect the weak dependence of $\delta \nu_{n, \ell}^{p}$ on $\ell$ (e.g., Monteiro et al. 1994; Houdek \& Gough 2007) when subtracting the frequencies of nearly degenerate modes. With these assumptions, the scaled small separations $D_{\ell, \ell+2} \equiv\left(\nu_{n, \ell}-\nu_{n-1, \ell+2}\right) /(4 \ell+6)$ for pairs of modes with degree $\ell \leq 3$ divided by the large separations $\Delta \nu_{n, \ell} \equiv \nu_{n+1, \ell}-\nu_{n, \ell}$ take the form

$$
\begin{aligned}
\frac{D_{02}}{\Delta \nu_{n-1,1}} & \approx \frac{\nu_{n, 0}^{s}-\nu_{n-1,2}^{s}}{6 \Delta \nu_{n-1,1}}+\frac{\delta \nu_{n, 0}^{c}}{6 \Delta \nu_{n-1,1}}, \\
\frac{D_{13}}{\Delta \nu_{n, 0}} & \approx \frac{\nu_{n, 1}^{s}-\nu_{n-1,3}^{s}}{10 \Delta \nu_{n, 0}} .
\end{aligned}
$$

In the high-frequency asymptotic regime, the quantity

$$
D_{\delta} \equiv \frac{\pi}{4 \ell+6} \frac{\nu_{n, \ell}^{s}-\nu_{n-1, \ell+2}^{s}}{\Delta \nu_{n, \ell}}
$$

in the unperturbed model is essentially independent of the pair of modes considered $(\ell=0,2$ or $\ell=1,3$; see Roxburgh \& Vorontsov 2000b a, 2003). Thus, we proceed by subtracting Eq. (13) from Eq. (12) to find the relation

$$
\frac{D_{02}}{\Delta \nu_{n-1,1}}-\frac{D_{13}}{\Delta \nu_{n, 0}} \approx \frac{\delta \nu_{n, 0}^{c}}{6 \Delta \nu_{n-1,1}} .
$$

Note that for the large separations appearing in the denominators, we used pairs of modes that encompass the frequency range of the modes used in the scaled small separations, which appear in the numerators. When defined in this way, the frequency differences presented in Eq. (15) are a smoother function of frequency than when $\Delta \nu_{n, 0}$ and $\Delta \nu_{n, 1}$ are used in the denominators of the first and second term, respectively. In practice, this means that when subtracting Eq. (13) from Eq. (12) we also had to assume that $\frac{\Delta \nu_{n, 0}}{\Delta \nu_{n-1,1}} \approx \frac{\Delta \nu_{n, 1}}{\Delta \nu_{n, 0}}$.

The frequency perturbation $\delta \nu_{n, 0}^{c}$ is expected to be modulated with frequency according to Eq. (10). Thus, if the assumptions leading to Eq. (15) hold, we would expect to see a corresponding modulation when the oscillation frequencies observed in a given solar-like pulsator are combined in the manner prescribed. In the next section we test this prediction on simulated data and we discuss what might be inferred about solar-like stars from this diagnostic tool, once observations with the necessary precision become available.

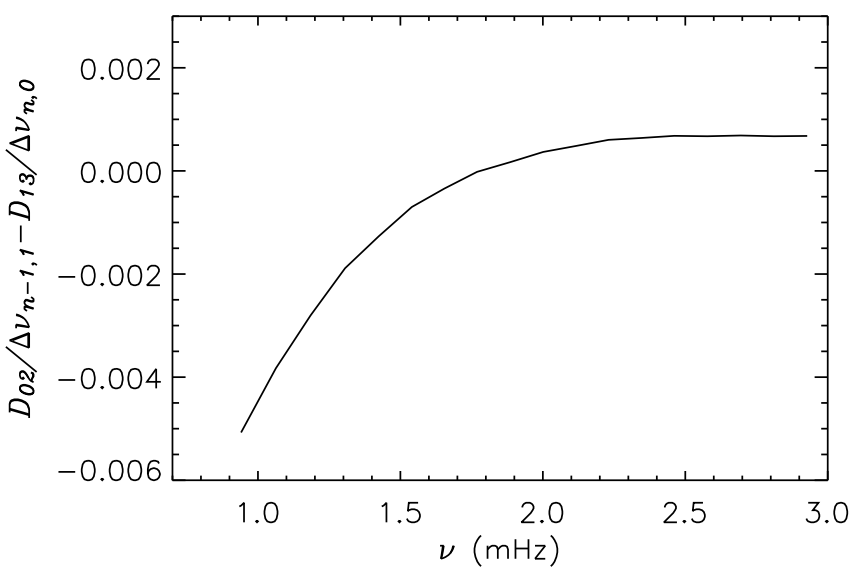

FIG. 3.- Frequency differences, as defined by the left hand side of Eq. (15), for a model with $M=1.3 \mathrm{M}_{\odot}$ at an age of $0.25 \mathrm{Gyr}$ when its convective core is very small and there is no discontinuity in the sound speed (see the top curve in Figure 1 .

\section{TESTS WITH SIMULATED DATA}

\subsection{Models}

We used the Aarhus stellar evolution code (ASTEC; Christensen-Dalsgaard 1982) coupled with the adiabatic pulsation code (ADIPLS) to calculate the theoretical oscillation frequencies for $1.3 \mathrm{M}_{\odot}$ models with ages from 0.25 to $5.25 \mathrm{Gyr}$, sampling the full range of convective core sizes along the evolution track. The ASTEC code used the equation of state (EOS) of Eggleton et al. (1973) without Coulomb corrections, and opacities from the OPAL tables (Iglesias \& Rogers 1996), supplemented by Kurucz opacities at low temperatures. The nuclear reaction rates came from Bahcall \& Pinsonneault (1992), convection was described by the mixing-length theory of Böhm-Vitense (1958), and we did not include the effects of diffusion.

We set the initial metallicity to $Z_{0}=0.02$, and the initial hydrogen mass fraction to $X_{0}=0.74$. We fixed the mixinglength parameter at $\alpha=1.9$ and included core overshoot using $\alpha_{\mathrm{ov}}=0.25$ with complete mixing in the overshoot region and considering the associated changes to the adiabatic temperature gradient. For each selected model, we calculated the radial and non-radial p-mode frequencies for oscillations with spherical degree $\ell=0-3$ and radial order $n=1 \sim 30$. We used these frequencies to construct the combinations shown in Eq. 15), and compared them to the theoretical predictions.

\subsection{Testing the diagnostic tool}

A number of approximations were made when deriving the diagnostic tool proposed in Eq. (15). Thus, it is essential that we evaluate the ability of this seismic tool when it comes to isolating the signal from the convective core.

With this in mind, we start by testing the last assumption made when deriving Eq. (15) — namely, the weak dependence of the value of $D_{\delta}$ in the unperturbed model on the pair of modes considered. To this end, we compute eigenfrequencies for our youngest model. At an age of $0.25 \mathrm{Gyr}$ the convective core is extremely small, and there is no apparent discontinuity in the sound speed (see the top curve in Figure 1). Under these conditions, we expect $\delta \nu_{n, 0}^{c} \approx 0$. Consequently, if the quantity $D_{\delta}$ were strictly independent of the pair of modes considered, the frequency differences defined by the expression on the left hand side (LHS) of Eq. (15) would be approximately zero for 

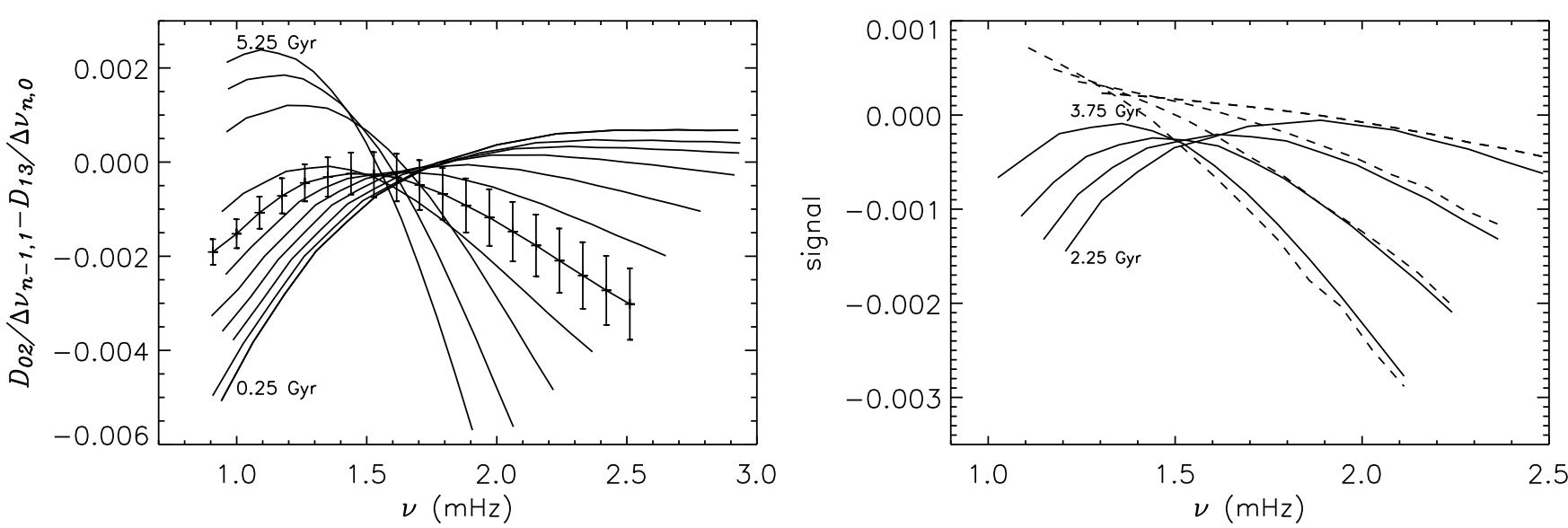

FIG. 4. - Left panel: the same as Figure 3 but for models with ages ranging from 0.25 Gyr (bottom left) to 5.25 Gyr (top left) in intervals of 0.5 Gyr. Error bars are shown for the model with maximum convective core size. These correspond to $1 \sigma$ errors in the frequency differences when the relative error in the individual frequencies is assumed to be $10^{-4}$. For this model, the radial order of the modes spanning the frequency range considered varies between $n=9$ and $n=27$. Right panel: comparison between the theoretical signal expected from the convective core (dashed lines) and that contained in the frequency differences (full curves) for models with ages ranging from $2.25 \mathrm{Gyr}$ (bottom left) to $3.75 \mathrm{Gyr}$ (top left) in intervals of $0.5 \mathrm{Gyr}$. To determine the expected signal, we computed $\delta \nu_{n, 0}^{\mathrm{c}}$ using Eq. [10] and then normalized it by the large separations to reproduce the right hand side of Eq. 15 .

\section{all frequencies.}

Figure 3 shows the frequency differences obtained for our youngest model. Clearly, in the lower frequency domain the frequency differences deviate significantly from zero. This reflects the fact that $D_{\delta}$ is nearly independent of the pair of modes considered only in the high-frequency asymptotic regime. At lower frequencies this assumption breaks down, and consequently so does the approximation made when deriving Eq. (15) from Eqs. (12) and (13). Nevertheless, at high frequencies $(\gtrsim 2 \mathrm{mHz}$ in Figure 3 ) the frequency differences converge to a constant small value, which we shall designate $\nu_{\text {asymp }}$, confirming our assumption of the near degeneracy of $D_{\delta}$ with respect to the pairs of modes $\ell=0,2$ and $\ell=1,3$.

To compare these results with those obtained for older models, we determine the frequency differences defined by the LHS of Eq. (15) for our sequence of models, with ages ranging from $0.25 \mathrm{Gyr}$ to $5.25 \mathrm{Gyr}$. The results are shown in the left panel of Figure 4 It is clear from this figure that in the high-frequency domain, where Eq. (15) is expected to be valid, the absolute value of the slope of the frequency differences increases with age. This can be understood as a direct consequence of the increase with age of the sound speed discontinuity at the edge of the convective core. According to Eq. (10), such an increase manifests itself through the amplitude $A_{\delta}$ by an increase in the absolute value of the frequency perturbation at fixed frequency. This increase is consequently reflected in the frequency differences defined by the LHS of Eq. (15), and thus in the slope of the curves plotted in Figure 4

To determine whether the signature of the convective core on the frequency differences might be detected in future observations of solar-like pulsators, we have included for one of our models the expected $1 \sigma$ error bars for the frequency differences, calculated with the assumption that the relative error on the individual frequencies is $10^{-4}$ (e.g., $0.2 \mu \mathrm{Hz}$ at $2 \mathrm{mHz}$ as expected from CoRoT; Baglin et al. 2006). It is evident from the figure that if individual frequencies are indeed determined with such precision, we will be able to not only detect the effect of the convective core on the frequencies, but also distinguish between different possible models based on this signature. Moreover, we see that our ability to detect this signature in real data will depend on the number of radial modes detected, as well as on their radial order.

We made additional assumptions, other than the one referred to at the beginning of this section, while deriving Eq. (15). In particular, we assumed that the effect of the convective core on the frequencies of modes with degree $\ell \geq 1$ is negligible, when compared to the effect on the frequencies of radial modes. To check whether this assumption is valid, we compare the frequency differences calculated for a subset of our models with the theoretical expectations derived from Eq. (10). The results are shown in the right panel of Figure 4 We see from the figure that in the high-frequency asymptotic regime the theoretical signal derived from the analysis of the wave equation near the inner turning point is indeed isolated when the oscillation frequencies are combined in the way suggested by Eq. (15). We note that there is no model dependent free parameter in the theoretical expression for the frequency perturbations. The difference in the slopes of the theoretical curves at high-frequency, seen also in the frequency differences calculated from simulated data, is thus a direct consequence of the dependence of the amplitude $A_{\delta}$ on the increase in the sound speed at the edge of the convective core.

In contrast to these results, Figure 4 shows that in the lowfrequency domain the combination of frequencies suggested by Eq. (15) does not properly isolate the theoretical signature expected from Eq. (10). Just as in the case discussed for Figure 3, this is likely a consequence of the breakdown at low frequencies of the assumption that $D_{\delta}$ is independent of the pair of modes considered. According to Figure 3 , when the frequency perturbation associated with the convective core is negligible, the frequency differences become negative in the low-frequency domain, decreasing as the frequency decreases. On the other hand, the frequency perturbation associated with the convective core becomes positive for frequencies below a particular value - namely, the frequency at which the position of the discontinuity in the sound speed coincides with the inner turning point of Eq. (2). In the low-frequency domain, we thus expect our diagnostic tool to show a superposition of these two behaviors. Indeed, this is seen for frequen- 

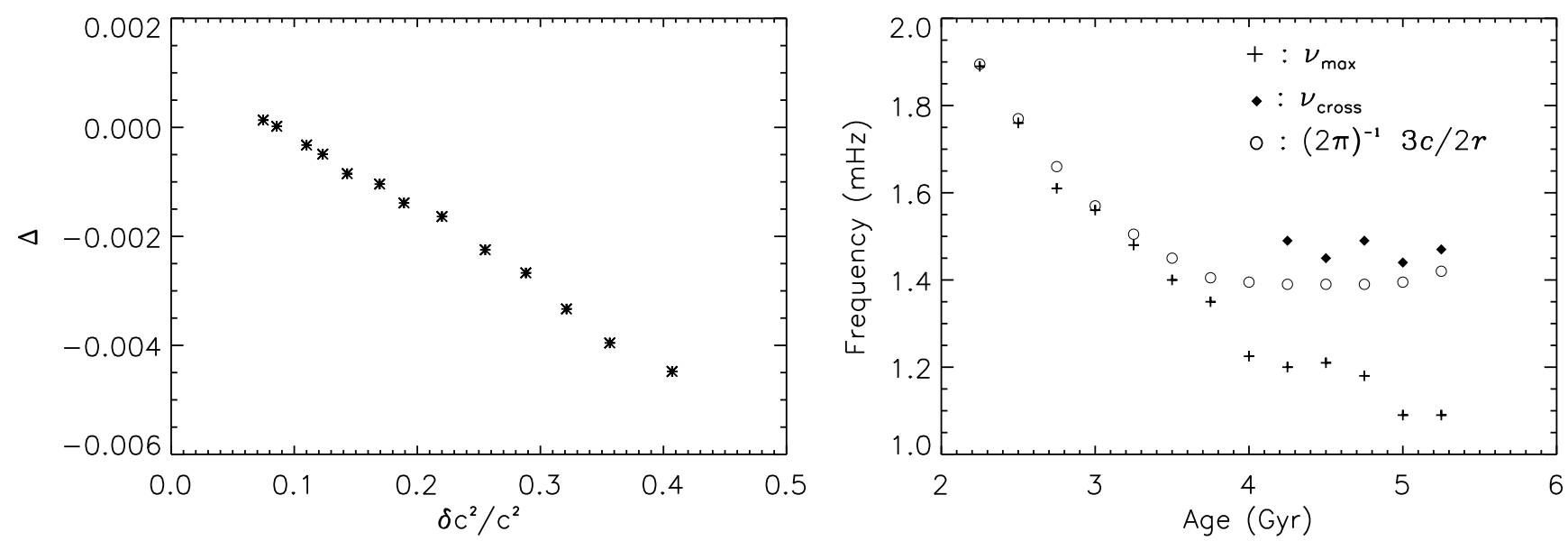

FIG. 5.- Left panel: measurement of the slope of the frequency differences in the high-frequency domain as a function of the relative increase in the squared sound speed at the edge of the convective core (see text for the mathematical definition of $\Delta$ ). The ages of the models shown range from $2.25 \mathrm{Gyr}$ (top left) to $5.25 \mathrm{Gyr}$ (bottom right) in intervals of $0.25 \mathrm{Gyr}$. Right panel: for models with ages ranging from $2.25 \mathrm{Gyr}$ to $5.25 \mathrm{Gyr}$, we show the frequency $\nu_{\text {max }}$ at which the frequency differences suggested by the LHS of Eq. [15) reach their maximum (crosses), and for the same models the value of $(2 \pi)^{-1} 3 c / 2 r$ (circles) at the edge of the convective core. Since $c$ is discontinuous at the edge of the convective core, we use the mean of the values of $c$ on the left and right side of the discontinuity when calculating this expression. For models with ages greater than $4 \mathrm{Gyr}$, we show the frequency $\nu_{\text {cross }}$ at which the frequency differences cross the asymptotic value for the youngest model (diamonds).

cies smaller than $\sim 1.5 \mathrm{mHz}$ in the left panel of Figure 4, The competition between these two behaviors results in the presence of a maximum in the frequency differences calculated for each model.

\subsection{Characterizing the convective core}

The validation of the diagnostic tool proposed in Eq. (15) in the high-frequency domain makes us confident that we understand the origin of the signature observed in the simulated data, and that we can use this signature to extract information about the convective core. As mentioned in $\$ 2.1$ and $\$ 3.2$ information about the size of the sound speed discontinuity at the edge of the convective core can be extracted from the amplitude of the signal, and in principle information about the ratio $[c / r]_{\text {edge }}$ at the edge of the convective core can be extracted from the value of the frequency at which the frequency perturbation goes through zero. Moreover, given the age dependence of the size of the sound speed discontinuity at the edge of the core, the signature discussed above might also be used to infer the evolutionary status of pulsating stars within the region of the H-R diagram considered here.

These ideas are illustrated in Figure 5. In the left panel the quantity $\Delta$, defined as the difference between the frequency differences $\left(\frac{D_{02}}{\Delta \nu_{n-1,1}}-\frac{D_{13}}{\Delta \nu_{n, 0}}\right)$ of modes with radial orders $n=k+2$ and $n=k-2$, with $k$ such that $\nu_{k} \sim 1750 \mu \mathrm{Hz}$ for each model, is shown as a function of the relative increase in the squared sound speed at the edge of the convective core. The quantity $\Delta$ provides a measure of the slope of the frequency difference curves, such as those plotted in the left panel of Figure 4 in the high-frequency domain. It is clear from Figure 5 that a linear relation exists between $\Delta$ and the squared sound speed increase at the edge of the convective core. We also considered alternative quantities indicative of the slope of the same curves taken at constant radial order rather than at constant frequency. In all cases the results indicate a linear relation with the squared sound speed increase at the edge of the convective core. Note that when studying data with noise, the use of the quantity $\Delta$ defined by only two data points is not advisable. Instead, it would be more conve- nient to extract the slope of the frequency difference curves in the high-frequency domain through a least-squares fit to the appropriate part of the curve under consideration.

Since the proposed diagnostic tool fails to reproduce the theoretical signal in the low-frequency domain, the idea of using this tool to determine the value of the frequency at which the frequency perturbation associated with the convective core goes through zero- and thus extracting information about the physical size of the core-becomes compromised. Nevertheless, since the maxima that characterize the curves defined by the frequency differences are a consequence of the positive frequency perturbations in the low-frequency domain, we might expect the position of these maxima to contain information about the size of the convective core. With this in mind, we have determined for each model the frequency $\nu_{\max }$ at which the frequency differences defined by the LHS of Eq. (15) reach their maximum value. These are plotted as a function of age in the right panel of Figure 5, and are compared with the frequencies $\left[(2 \pi)^{-1} 3 c / 2 r\right]_{\text {edge }}$ at which the frequency perturbation associated with the convective core goes through zero.

Clearly, the frequency at which the frequency differences reach their maxima, indicated by the crosses in Figure 5, is strongly correlated with the age of the model. This is not surprising since the magnitudes of the frequency perturbations, which influence the position of the maxima, are also correlated with age. Nevertheless, a change in the slope is noticeable around an age of $\sim 4$ Gyr. This is likely a consequence of the contracting core. As seen in Figure 1, when the core begins to contract, the sound speed variation near the edge of the convective core begins to spread over a larger radial extent. Our theoretical analysis, which assumes that the sound speed variation at the edge of the core is well represented by a modified step function, becomes less adequate in this case. Nevertheless, an inspection of the frequency differences shown in the left panel of Figure 4 indicates that in the older models where this effect becomes evident, the amplitude of the frequency differences in the low-frequency domain is indeed substantially larger than in all other mod- 
els. This may result from a sudden increase in the amplitude of the frequency perturbation in the low-frequency domain, or from an improvement in the degeneracy of $D_{\delta}$ calculated for pairs of modes with degrees $\ell=0,2$ and $\ell=1,3$. Whatever the case, the relative importance of the latter in determining the functional form of the frequency differences appears to be smaller for models with ages greater than $\sim 4$ Gyr. Thus, one might expect that for these models the frequency $\left[(2 \pi)^{-1} 3 c / 2 r\right]_{\text {edge }}$ will be better estimated by the frequency $\nu_{\text {cross }}$ at which the frequency differences cross the asymptotic value for the youngest model in Figure 3. Indeed this seems to be the case, as shown by the diamonds in the right panel of Figure 5

\section{DISCUSSION}

We have derived the expected signature of small convective cores on the oscillation frequencies of solar-like pulsators, we have suggested a diagnostic tool to isolate this signature, and finally we have discussed what information the signature may reveal about the convective cores themselves and about the evolutionary status of the corresponding stars.

The results demonstrate that our ability to isolate the convective core signature with the suggested diagnostic tool is significantly better in the high-frequency asymptotic domain than at lower frequencies. Consequently, the information inferred from this diagnostic tool at high frequencies is expected to be more robust. In particular, this is the case for the relation found between the slope of the frequency difference curves and the relative increase in the squared sound speed at the edge of the convective core. Based on the theoretical signal, which in the high-frequency domain is well represented by the proposed diagnostic tool, we expect this relation to hold also in a more general context when models of different mass, chemical composition, or with different amounts of core overshoot are considered.

By contrast, information inferred from the behaviour of the diagnostic tool at lower frequencies, such as the estimation of the ratio $[c / r]_{\text {edge }}$ from the position of the maximum in the frequency differences, or from the value of the frequency at which these differences cross $\nu_{\text {asymp }}$, is likely to show a stronger model dependence. As emphasized earlier, at lower frequencies the functional form of the diagnostic tool is influenced by the deviation from degeneracy of the quantity $D_{\delta}$. Consequently, this tool does not capture, as well as it does at higher frequencies, the effect of the convective core on the oscillations, even though it is still influenced by it. Therefore, it is important to extend the present study to different models, varying quantities such as mass, chemical composition, and core overshoot, to determine the extent to which the quantities studied-particularly in the low-frequency domain-depend on the properties of the models.

One aspect of major importance that has been considered by most works involving diagnostic tools to study the inner regions of stars is the ability of these tools to determine the stellar age. The correlations between the quantities studied in $\$ 3$ and the age of the models are clear. Hence, in principle the former might be used to infer the evolutionary status of pulsating stars. In fact, this correlation is responsible for the one found by Mazumdar et al. (2006) between their quantity $\theta$ and the central hydrogen abundance $X_{c}$, (cf. figure 7 of their paper). Their $\theta$ is proportional to the difference $\left(D_{13}-D_{02}\right)$ averaged over several radial orders $n$, and through $X_{c}$ it reflects the change in the slope seen in the left panel of our Figure 4 Thus, our analysis also provides the theoretical basis for the correlation found by Mazumdar et al. (2006). Nevertheless, it is important to investigate how these correlations depend on other stellar parameters, and to ascertain whether there are degeneracies in the age determination when the appropriate parameter space is explored. Also, as mentioned in $\S 3.1$ the models used to test our diagnostic tool did not include diffusion. The presence of diffusion will likely result in a smoother transition in $c^{2}$. We hope to tackle the consequences of including diffusion in future work.

Asteroseismic data with the precision necessary to test our diagnostic tool observationally are soon expected from the CoRoT satellite (Baglin et al. 2006). The second scheduled 5-month run will include the star HD 49933, which exhibits solar-like oscillations (Mosser et al. 2005) and has approximately the required mass and age to reveal the expected signal. Future observations of the slightly more massive spectroscopic binary star 12 Boötis (Miglio et al. 2007) could also provide an interesting test of our predictions.

The authors are very grateful to Jørgen ChristensenDalsgaard and Douglas Gough for helpful comments and suggestions. This work was supported in part by the EC's FP6, FCT and FEDER (POCI2010) through the HELAS international collaboration and through the project POCI/CTEAST/57610/2004, by a Fulbright grant under the Mutual Educational Exchange Program, and by NCAR through the ECSA and HAO Visiting Scientist Programs. The National Center for Atmospheric Research is a federally funded research and development center sponsored by the U.S. National Science Foundation.

\section{APPENDIX}

\section{A. SOLUTION TO THE WAVE EQUATION NEAR THE INNER TURNING POINT}

In this appendix we derive an approximate solution to Eq. (2), valid near the inner turning point $z_{1}$ at which $K^{2}=0$. We closely follow the derivation presented in $\$ 4.8 .5$ of Gough (1993), though the dependent and independent variables in our Eq. (2) differ from those in Gough's Eq. (4.8.5).

We start by defining new independent and dependent variables, $x$ and $\psi$ respectively

$$
\begin{aligned}
& x=\operatorname{sgn}\left(\mathrm{K}^{2}\right)\left[\operatorname{sgn}\left(\mathrm{K}^{2}\right) \frac{3}{2} \int_{z_{1}}^{z}|K| \mathrm{d} z\right]^{\frac{2}{3}}, \\
& \psi=|x|^{-\frac{1}{4}}|K|^{\frac{1}{2}} \Xi \equiv s^{-\frac{1}{2}} \Xi .
\end{aligned}
$$

With these definitions, $x=0$ at the inner turning point. Substituting these into Eq. (2) we find

$$
\frac{\mathrm{d}^{2} \psi}{\mathrm{d} x^{2}}+x \psi=-s^{\frac{3}{2}} \frac{\mathrm{d}^{2} s^{\frac{1}{2}}}{\mathrm{~d} z^{2}} \psi
$$


where we keep the independent variable $z$ in the right hand side (RHS) to keep the equation in a condensed form.

To find an asymptotic solution to Eq. (A3) we regard the term on the RHS as a small perturbation to the Airy equation (Olver 1974). We note that our original transformation of the independent variable $z=\ln \left(r / r_{\text {ref }}\right)$, was motivated precisely by the need to keep the RHS bounded when $r$ tends to zero (Langer 1937). In fact, a straightforward analysis of this term shows that it will tend to zero as $r$ tends to zero.

To leading order, we thus have that the solution to Eq. (A3) around the inner turning point can be obtained from

$$
\psi \approx a A_{i}(-x)+b B_{i}(-x)
$$

where $A_{i}$ and $B_{i}$ are the solutions to the Airy equation, and $a$ and $b$ are constants. Since the solution must tend to zero as $x$ tends to $-\infty$, we must have $b=0$. Using the ascending series for $A_{i}$ (e.g. Abramowitz \& Stegun 1972, 110.4 , p.446), we thus find for $|x| \ll 1$

where

$$
\psi \approx a\left[A_{i}(0)-A_{i}^{\prime}(0) x+\mathcal{O}\left(x^{3}\right)\right],
$$

and $\Gamma$ is the Gamma function. Transforming back, we then find that

$$
A_{i}(0)=\frac{1}{3^{\frac{2}{3}} \Gamma\left(\frac{2}{3}\right)} \text { and } A_{i}^{\prime}(0)=-\frac{1}{3^{\frac{1}{3}} \Gamma\left(\frac{1}{3}\right)},
$$

with $s$ defined by Eq. (A2).

$$
\Xi \approx a s^{\frac{1}{2}}\left[A_{\mathrm{i}}(0)-A_{\mathrm{i}}^{\prime}(0) x\right],
$$

\section{B. VARIATIONAL ANALYSIS}

In this appendix we derive the expression for the frequency perturbations resulting from the presence of a small convective core. Our starting point is Eq. (7), with $\delta I_{1}$ and $\delta I_{2}$ defined by Eqs. (8) and (9). Introducing the new variables $x$ and $\psi$ into Eqs. (8) and (9) and noting that

we find, after some algebra,

$$
\left(\frac{\mathrm{d} \psi}{\mathrm{d} x}\right)^{2}=\frac{1}{2} \frac{\mathrm{d}^{2} \psi^{2}}{\mathrm{~d} x^{2}}-\psi \frac{\mathrm{d}^{2} \psi}{\mathrm{d} x^{2}} \text { and } \frac{\mathrm{d}^{2} \psi}{\mathrm{d} x^{2}} \approx-x \psi
$$

$$
2 I_{1} \omega \delta \omega \approx \int_{X_{0}}^{X_{R}}\left[f_{1} \psi^{2}+f_{2} \frac{\mathrm{d} \psi^{2}}{\mathrm{~d} x}+f_{3} \frac{\mathrm{d}^{2} \psi^{2}}{\mathrm{~d} x^{2}}\right] \mathrm{d} x,
$$

where $X_{0}$ and $X_{R}$ are the limits of the original integral in terms of the new variable $x$ and

$$
\begin{aligned}
& f_{1}=r^{3} q^{2}\left\{s^{-1}\left[x+\left(\frac{1}{q} \frac{\mathrm{d} q}{\mathrm{~d} x}\right)^{2}\right] \delta(\gamma p)-\frac{\mathrm{d}}{\mathrm{d} x}[3 \delta(\gamma p)-4 \delta p]-\omega^{2} r^{2} s \delta \rho\right\}, \\
& f_{2}=\frac{1}{2} r^{3} s^{-1} \frac{\mathrm{d} q^{2}}{\mathrm{~d} x} \delta(\gamma p), \\
& f_{3}=\frac{1}{2} r^{3} s^{-1} q^{2} \delta(\gamma p),
\end{aligned}
$$

with $q=s^{1 / 2} r^{-3 / 2} r_{\text {ref }}{ }^{-1 / 2} \rho^{-1 / 2} c^{-1}$.

Next, we express Eq. (B2) in terms of the perturbations $\delta(\gamma p) / \gamma p$ and $\delta c^{2} / c^{2}$. Using the relations

we find

$$
\frac{\delta \rho}{\rho}=\frac{\delta(\gamma p)}{\gamma p}-\frac{\delta c^{2}}{c^{2}} \text { and } \frac{\mathrm{d} \delta p}{\mathrm{~d} x}=-\rho g r s\left(\frac{\delta g}{g}+\frac{\delta(\gamma p)}{\gamma p}-\frac{\delta c^{2}}{c^{2}}\right),
$$

$$
2 I_{1} \omega \delta \omega \approx \int_{X_{0}}^{X_{R}}\left[\left(\delta \mathcal{F}_{1}+\frac{\mathrm{d}}{\mathrm{d} x}\left(\delta \mathcal{F}_{0}\right)\right) \psi^{2}+\delta \mathcal{F}_{2} \frac{\mathrm{d} \psi^{2}}{\mathrm{~d} x}+\delta \mathcal{F}_{3} \frac{\mathrm{d}^{2} \psi^{2}}{\mathrm{~d} x^{2}}\right] \mathrm{d} x,
$$

where

$$
\begin{aligned}
\delta \mathcal{F}_{0}= & -\left(3 r^{3} q^{2} \gamma p\right) \frac{\delta(\gamma p)}{\gamma p}, \\
\delta \mathcal{F}_{1}= & \left\{r^{3} q^{2}\left[s^{-1}\left(x+\left(\frac{1}{q} \frac{\mathrm{d} q}{\mathrm{~d} x}\right)^{2}\right) \gamma p-\rho r s\left(4 g+\omega^{2} r\right)\right]+3 \gamma p \frac{\mathrm{d}\left(r^{3} q^{2}\right)}{\mathrm{d} x}\right\} \frac{\delta(\gamma p)}{\gamma p} \\
& +\left[r^{4} q^{2} \rho s\left(4 g+\omega^{2} r\right)\right] \frac{\delta c^{2}}{c^{2}} \\
& -\left(4 r^{4} q^{2} \rho s g\right) \frac{\delta g}{g} \\
\delta \mathcal{F}_{2}= & \left(\frac{1}{2} r^{3} s^{-1} \frac{\mathrm{d} q^{2}}{\mathrm{~d} x} \gamma p\right) \frac{\delta(\gamma p)}{\gamma p}, \\
\delta \mathcal{F}_{3}= & \left(\frac{1}{2} r^{3} s^{-1} q^{2} \gamma p\right) \frac{\delta(\gamma p)}{\gamma p} .
\end{aligned}
$$


The perturbation associated with the discontinuity at the edge of the convective core is rather localized. Thus, we consider an interval $\left[X_{a}, X_{b}\right]$ in which the perturbation differs from zero and assume it to be equal to zero outside that interval. By requiring both that the sound speed is everywhere the same in the perturbed and unperturbed models except near the discontinuity, and that both models are in hydrostatic equilibrium, the small difference in their density profiles extends beyond the region of the discontinuity, towards the center of the star. Nevertheless, because we are interested in isolating the frequency perturbation associated with the rapid variation at the edge of the core, we do not consider the perturbation arising from this additional difference between the two models.

Integrating by parts all terms of the integral on the RHS of Eq. (B4) except for that involving $\delta \mathcal{F}_{0}$, we find

$$
2 I_{1} \omega \delta \omega \approx-\int_{X_{a}}^{X_{b}}\left[\left(\frac{\mathrm{d} \delta \mathcal{F}_{1}}{\mathrm{~d} x}\right) \overline{\psi^{2}}+\left(-\frac{\mathrm{d} \delta \mathcal{F}_{0}}{\mathrm{~d} x}+\frac{\mathrm{d} \delta \mathcal{F}_{2}}{\mathrm{~d} x}\right) \psi^{2}+\frac{\mathrm{d} \delta \mathcal{F}_{3}}{\mathrm{~d} x} \frac{\mathrm{d} \psi^{2}}{\mathrm{~d} x}\right] \mathrm{d} x,
$$

where $\overline{\psi^{2}}=\int \psi^{2} \mathrm{~d} x$.

At the edge of the convective core, $\delta c^{2} / c^{2}$ is discontinuous while all other perturbations in Eq. (B4) are not. Hence, the main contribution to the frequency perturbations will come from the term involving the perturbation to the sound speed. Accordingly, we neglect all other terms and find

$$
2 I_{1} \omega \delta \omega \approx-\int_{X_{a}}^{X_{b}} \frac{\mathrm{d}}{\mathrm{d} x}\left[r^{4} q^{2} \rho s\left(4 g+\omega^{2} r\right) \frac{\delta c^{2}}{c^{2}}\right] \overline{\psi^{2}} \mathrm{~d} x .
$$

We model the sharp variation $\delta c^{2} / c^{2}$ at the edge of the convective core, $x_{\mathrm{d}}$, with a function of the type $\delta c^{2} / c^{2} \propto\left[H\left(x-x_{\mathrm{d}}\right)-1 / 2\right]$, where $H$ is the Heaviside Step Function and the proportionality constant is determined by the amplitude of the sharp variation. Under this assumption, and keeping in mind that the term multiplying $\delta c^{2} / c^{2}$ in Eq. (B6) is a slowly varying function of $x$, we have

$$
\frac{\mathrm{d}}{\mathrm{d} x}\left[r^{4} q^{2} \rho s\left(4 g+\omega^{2} r\right) \frac{\delta c^{2}}{c^{2}}\right] \approx A_{\delta}\left[r^{4} q^{2} \rho s\left(4 g+\omega^{2} r\right)\right] \delta\left(x-x_{\mathrm{d}}\right),
$$

where $A_{\delta}$ is the amplitude associated with the $\delta$-function. We thus find, after some algebra,

$$
2 I_{1} \omega \delta \omega \approx-A_{\delta} a^{2} A_{i}(0)^{2}\left[\frac{r\left(4 g+\omega^{2} r\right)}{r_{\mathrm{ref}} c^{2}|K|^{2}}\left(|x| x-\frac{A_{\mathrm{i}}^{\prime}(0)}{A_{\mathrm{i}}(0)}|x| x^{2}+\frac{1}{3} \frac{A_{\mathrm{i}}^{\prime}(0)^{2}}{A_{\mathrm{i}}(0)^{2}}|x| x^{3}\right)\right]_{x=x_{\mathrm{d}}} .
$$

In practice, if the approximations introduced above are reasonably satisfied, $A_{\delta}$ is expected to be proportional to the amplitude of the sharp variation $\delta c^{2} / c^{2}$ at $x_{\mathrm{d}}$, with the proportionality constant independent of the model considered. Indeed we found this to be the case in all of our models except for those older than $\sim 4$ Gyr. For these models the discontinuity in $c^{2}$ is not as sharp as for younger models. Even so, the functional form of the perturbations was still found to be relatively well represented by Eq. (B8) in this case.

Finally, the constant $a$ can be expressed in terms of the amplitude of the eigenfunction $\xi$ at the inner turning point. Combining the definitions of $\psi$ and $\Xi$, we find

$$
[\psi]_{x=0}=\left[q^{-1} \xi\right]_{x=0}=a A_{i}(0) .
$$

Substituting this expression for $a$ into Eq. (B8), we finally have

$$
\delta \omega \approx-\frac{A_{\delta}}{2 \omega I_{1}}\left[\frac{|K| r^{3} \rho c^{2} \xi^{2}}{|x|^{1 / 2}}\right]_{x=0}\left[\frac{r\left(4 g+\omega^{2} r\right)}{c^{2}|K|^{2}}\right]_{x=x_{\mathrm{d}}}\left[|x| x-\frac{A_{\mathrm{i}}^{\prime}(0)}{A_{\mathrm{i}}(0)}|x| x^{2}+\frac{1}{3} \frac{A_{\mathrm{i}}^{\prime}(0)^{2}}{A_{\mathrm{i}}(0)^{2}}|x| x^{3}\right]_{x=x_{\mathrm{d}}} .
$$

For practical reasons, when calculating the theoretical frequency perturbations from Eq. (B10), we use the quantities $\omega, I_{1}$ and $\xi_{x=0}$ calculated for the model with the discontinuity. To first order, this should have no impact on the theoretical signal.

\section{REFERENCES}

Abramowitz, M. \& Stegun, I. A. 1972, Handbook of Mathematical Functions (9th printing, New York: Dover)

Aerts, C., et al. 2006, ApJ, 642, L165

Baglin, A., et al. 2006, ESA SP-624: Proceedings of SOHO 18/GONG 2006/HELAS I, Beyond the spherical Sun, 18

Bahcall, J. N., \& Pinsonneault, M. H. 1992, ApJ, 395, L119

Basu, S. 1997, MNRAS, 288, 572

Bedding, T. R., et al. 2005, A\&A, 432, L43

Böhm-Vitense, E. 1958, Zeitschrift fur Astrophysik, 46, 108

Bruntt, H., et al. 2007, A\&A, 461, 619

Buzasi, D., Catanzarite, J., Laher, R., Conrow, T., Shupe, D., Gautier, T. N., III, Kreidl, T., \& Everett, D. 2000, ApJ, 532, L133

Cameron, C., et al. 2007, Communications in Asteroseismology, in press (astro-ph/0610822)

Chandrasekhar, S. 1964, ApJ, 139, 664

Christensen-Dalsgaard, J. 1982, MNRAS, 199, 735
Christensen-Dalsgaard, J., Arentoft, T., Brown, T. M., Gilliland, R. L., Kjeldsen, H., Borucki, W. J., \& Koch, D. 2007, Communications in Asteroseismology, in press (astro-ph/0701323)

Cox, J. P. 1980, Theory of stellar pulsation (Princeton, NJ: Princeton University Press)

Dappen, W., Dziembowski, W. A., \& Sienkiewicz, R. 1988, IAU Symp. 123: Advances in Helio- and Asteroseismology, 123, 233

Eggleton, P. P., Faulkner, J., \& Flannery, B. P. 1973, A\&A, 23, 325

Gough, D. O. 1993, in Astrophysical fluid dynamics, eds. J-P. Zahn \& J. Zinn-Justin (Amsterdam: Elsevier), 399

Houdek, G. \& Gough, D.O. 2007, MNRAS, 375, 861

Iglesias, C. A., \& Rogers, F. J. 1996, ApJ, 464, 943

Langer, R.E. 1937, Phys. Rev., 51, 669

Matthews, J. M., Kusching, R., Guenther, D. B., Walker, G. A. H., Moffat, A. F. J., Rucinski, S. M., Sasselov, D., \& Weiss, W. W. 2004, Nature, 430, 51 
Mazumdar, A., Basu, S., Collier, B. L., \& Demarque, P. 2006, MNRAS, 372, 949

Miglio, A., Montalban, J., \& Maceroni, C. 2007, MNRAS, 377, 373

Monteiro, M. J. P. F. G., Christensen-Dalsgaard, J., \& Thompson, M. J. 1994 A\&A, 283, 247

Monteiro, M. J. P. F. G., Christensen-Dalsgaard, J., \& Thompson, M. J. 2000, MNRAS, 316, 165

Monteiro, M. J. P. F. G., \& Thompson, M. J. 1998, IAU Symp. 185: New Eyes to See Inside the Sun and Stars, 185, 317

Monteiro, M. J. P. F. G., \& Thompson, M. J. 2005, MNRAS, 361, 1187

Mosser, B., et al. 2005, A\&A, 431, L13

Olver, F. W. J. 1974, Asymptotics and special functions (New York: Academic Press)
Randall, S. K., et al. 2005, ApJ, 633, 460

Rowe, J. F., et al. 2007, Communications in Asteroseismology, in press (astro-ph/0610640)

Roxburgh, I. W., \& Vorontsov, S. V. 2000a, MNRAS, 317, 141

Roxburgh, I. W., \& Vorontsov, S. V. 2000b, MNRAS, 317, 151

Roxburgh, I. W., \& Vorontsov, S. V. 2001, MNRAS, 322, 85

Roxburgh, I. W., \& Vorontsov, S. V. 2003, A\&A, 411, 215

Saio, H., et al. 2006, ApJ, 650, 1111

Takata, M., 2006, PASJ, 58, 893

Walker, G., et al. 2003, PASP, 115, 1023 